\title{
An Efficient Proposed Approach of Dynamic Clustering for Target Tracing in Wireless Sensor Networks
}

\author{
Manas Kumar Ray ${ }^{1}$ and Gitanjali Roy ${ }^{2}$ \\ Lecturer, Department of Computer Application, Banwarilal Bhalotia College, Asansol, West Bengal, India \\ Lecturer, Department of Computer Application, Bidhan Chandra College, Asansol, West Bengal, India \\ E-Mail: manasray12@gmail.com, groyasn@gmail.com
}

\begin{abstract}
This paper proposes and evaluates decentralized dynamic clustering algorithm for tracing a movable target. Here firstly we proposed dynamic $K$ mean clustering algorithm. In this algorithm a fixed number of sensor nodes is choose and then cluster is created. When the cluster is created then a cluster head (CH) is active. This active $\mathbf{C H}$ sensor nodes will create new cluster and that new cluster is also formed a new mean value of cluster head. But, the newly created cluster is only active when a moving objected is trace. According from the position of cluster head, few sensor nodes is active, where as few sensor nodes are inactive. According from the $\mathbf{C H}$ nodes newly cluster is created. So, creation of dynamic cluster is less energy efficient and stability of cluster will more than static cluster with sensor nodes. On the other hand, movable object tracing sensor nodes are familiar with energy utilization of sensor nodes. Here we proposed an energy efficient target tracing approach which follow network stability as well as energy saving. As we use dynamic clustering technique, so optimization of energy each sensor nodes with cluster head is maximum. So all the sensors with cluster head sensor nodes will continue more time for object tracing. In simulation result we show that our proposed dynamic $K$ mean clustering algorithm is more accurate and more stable.
\end{abstract}

Keywords: Wireless Sensor Network (WSN), Dynamic Clustering, Cluster Head (CH), K Mean Clustering

\section{INTRODUCTION}

Now-a-day's cluster analysis is an important task in research. It's aim is to analysis of data as make a group either similarity or dissimilarity. The analysis of data within a cluster divided into two types one is supervised and unsupervised manner. Different approach can be applied in clustering in data. But $\mathrm{K}$ mean clustering is one and different on them. Because here we proposed a dynamic cluster technique and within that cluster we locate a movable object. So tracing of object is also an important task within that dynamic cluster. In clustering technique most algorithms take a fixed number of clusters. In real world it is very difficult task to make cluster with unknown domain data set. In this paper we applied modified $\mathrm{K}$ mean dynamic clustering technique. Actually $\mathrm{K}$ mean algorithm run in two ways. Firstly number of cluster is fixed and secondly the number of cluster is not fixed, it cans change. If the number of cluster is fixed then it will run successfully as $\mathrm{K}$ mean clustering where as if the number of cluster is not fixed by user, then it is fixed by a random number. Beyond the random number $\mathrm{K}$ mean produces cluster and increment the number of cluster one by one.
Generally K mean clustering is a heuristic approach. First it takes the distance from all samples and then it minimizes the sum of squares and also $\mathrm{K}$ means algorithm find the number of clusters. The number of clusters is predefined. But the fixed number of cluster form a poor cluster where as $\mathrm{K}$ mean algorithm with random generated number create strong clusters. Here we proposed an approach with random generated number of $\mathrm{K}$ mean clusters.

Dynamic cluster architecture has several features. In wireless sensor network the cluster are forms in several events. Such as detection of target with wireless sensor network using acoustic sound, radio wave frequency etc. Tracing a movable target has various approaches. Firstly cluster head $(\mathrm{CH})$ take the all the position of all sensor nodes and then cluster head check its databases and update the sensors location if any movable object come in that cluster. Secondly all the sensor nodes form a cluster according to their cluster head position and the formation of cluster is changed if any movable object will come. To do the data processing in target tracing in wireless sensor network, the cluster architecture plays an important role. Actually the sensor nodes within the cluster have cluster head and many neighboring sensor nodes. In general cluster architecture cluster are formed statically when the network is deployed. So the parameter like coverage of cluster, timing of clusters, and implementation of clusters are all static.

According to the deployment of sensor nodes technique the cluster is mainly divided into two parts that is static cluster and dynamic clusters. Implementation of static cluster is simple. But beyond the simplicity of static cluster, it has several drawbacks. As in static cluster is fixed implementation of sensor node deployment, it cannot replace fault sensor nodes. And also if cluster head sensor node dies, then whole the cluster within that cluster head position also disable. If a cluster head is dies then within that cluster may not have sufficient sensor nodes to replace that cluster nodes. On the other hand, dynamic cluster has several features. Formation of dynamic clusters is completed by several events. In our proposed approach the formation of dynamic cluster is triggered by detection of movable object. It is a decentralized approach, so no excessive message exchange is required. As a result in dynamic cluster sensors will have more time to track the movable object. And also in dynamic clustering method 
only one cluster head is active at a time. Beyond the cluster head within the cluster that sensor nodes will active and else other nodes will stand by mode. So it is a less energy efficient and will track more time for movable object.

In this paper we evaluate a decentralized dynamic clustering algorithm. We also focus on the target of movable single object location. The rest of the paper is organized as follows: we show Related Work in section 2. We depict our Proposed Approach in section 3. In section 4 describe our Simulation Results. After that we analysis our Simulation Result. Lastly we show the Conclusion and Future Work in Section 5 and Section 6 respectively.

\section{REVIEW OF RELATED WORKS}

S. Prakash kumar and K. S. Ramaswami describe K mean clustering approach [1]. It is a heuristic approach. It can maximize the sum of squires of distance. Here all samples merging in cluster domain as the objective function of $\mathrm{K}$ mean cluster seek to minimum number of cluster. It is a standard and efficient approach. The authors describe as $\mathrm{K}$ input is accepted and n number of data objects is selected. $\mathrm{K}$ is a cluster domain i.e. cluster domain into $\mathrm{K}$ types. $\mathrm{K}$ is selected randomly and is used to cluster observation into groups without any previous knowledge of data. So that cluster technique will make group without any prior planning. It is commonly used in biometrics, sensors etc. In [2], Jiawei Han and Micheline Kamber, describe a partitioning method. Here $\mathrm{K}$ is a number of partitions of data where each partition represents clusters. In this approach author represent some conditions. The conditions are at least one objects must be exits in a particular group. Then each group exactly is belongs through an object. Here authors have investigated many features of $\mathrm{K}$ mean clusters. The performance of $\mathrm{K}$ mean factor may affect various performances.

In [3][4] authors describe $\mathrm{K}$ mean algorithm which is commonly used partition clustering algorithm. It is easy to implement and is the most efficient of execution time. Here the authors mainly describe about coverage of area. The coverage is basically local optimum and clustering technique is easy and less time consuming. Lastly it is proof that $\mathrm{K}$ mean clustering is efficient approach. Target tracing in wireless sensor network recently attract much attention. Actually the target tracing can be divided into two estimation technique. First measure the localization of sensor nodes of an area and secondly tracing the object location within that area. As in localization technique, every sensor nodes know its current position. The data captured through the sensor nodes are collected in base station. This signal processing scheme is divided into two types.

In [5] M. Chu, H. Haussecker and F. Zhao describe the signal processing scheme using maximum likely hood function and in [6] M. Chu, H. Haussecker and F. Zhao describe minimum square estimation for trace a moving object. In [7][8][9] authors describe the target location. It is estimated successfully based on current position at a sensor.
Initially the authors approach the previous position of object and according from initial position, the next position is measured. So that moving object easily traced. This approach is efficient for optimum energy estimation and easy to implement. In [10] Y. Xu and W.C. Lee and [11] G.Y. Jin, X.Y. Lu, and M.S. Park, the authors describe tracing mechanism of moving object with static clustering technique. In this technique all the sensor nodes are always activate to monitor an object. Due to continuous activation of all the sensor nodes, this technique is energy efficient technique and also time consuming.

W. Heinzelman, A. Chandrakasan, and H. Balakrishnan [12] describe a monitoring scheme of an object. In this approach some sensor nodes activate to monitor an object, instead of all the sensor nodes. Here one sensor node active to trace an object and then the neighbor sensor nodes will wake up. So it will keep constantly monitoring of a moving object where some sensor nodes wake up and some sensor nodes is standby mode. In this technique sensor nodes save energy, so it will monitor object long time. Here the author also describes the dynamic clustering technique. In this technique the cluster head get very strongest signal. This cluster head play a important role to make a dynamic cluster. This cluster head sent and receive data within the sensors, so dynamically trace moving object easily.

In Zhang, Y. Yang, L.T. Chen, J. RFID [13] describe localization technique of senor network. The authors divided the entire task in two category, range based and range free. In range based technique sensor nodes are deployed in such a way that the whole process is less time consuming and easy to implement. But in range free technique, the sensors are deployed randomly. It is less energy efficient but little time consuming. Patwari, N.; Ash, J.; Kyperountas, $\mathrm{S}$ describe the range free localization technique [14]. But here the sensor nodes are deployed using two parameters that are Received Signal Strength and Time of Arrival (ToA). In both parameters the approach proposed to determine the accuracy and energy estimation.

\section{PROPOSED APPROACH}

Actually $\mathrm{K}$ mean dynamic clustering is used for partitioning. It is a $\mathrm{K}$ number of clusters and $\mathrm{n}$ number of data sets. Generally $\mathrm{K}$ is choose randomly and data sets containing $\mathrm{n}$ objects. In each cluster all the sensors have similar pattern and take the mean value of each clusters. When new cluster is formed then update the mean value and it will repeat until it end of the entire area. But, our proposed dynamic clustering algorithm is composed of two parts. Firstly we describe dynamic clustering technique and then measure the location moving object through sensor nodes. As we proposed an approach of our $\mathrm{K}$ mean algorithm which divided into three parts, $\mathrm{K}$ mean algorithm improve cluster quality and fix the optimal number of clusters. Here, we describe part1 as Initialization, Assignment of Cluster predicts in Part2 and lastly in part3 we predict Measure of Centroid of Sensor nodes. Part1: initialization: Here we choose randomly $\mathrm{K}$ type sensors 
point location. Sensor point location take location point data randomly from all the sensors set. We proposed $\mathrm{K}$ types locations are as initial centroids and the initial centroid sensors measure the location of each sensor.Part2: Then, all the sensors location point to a centroid will create a cluster and we are using Euclidean distance between sensors location point and all the $\mathrm{K}$ type of centroid sensors. After that we take a straight line between two centroid sensors. These two centroid sensors choose randomly among from all $\mathrm{K}$ type. So that two cluster will create.Part3: After that, we take the mean value of each cluster. This mean value in centroid sensors among of newly two created clusters respectively. Next, we do the step2 and step3 until centroid sensors ends in moving. So, $K$ mean algorithm is completely covered. Tracking of an object: Here we used trilateration algorithm. Our proposed approach is taking the next location of target. Here we get the previous and present location of target and measure the current location of target. Here all the sensors are clustered with dynamic clustering method. We take three sensors which are anchor nodes, namely, A,B,C and their position are $\left(\mathrm{x}_{\mathrm{a}}, \mathrm{y}_{\mathrm{a}}\right),\left(\mathrm{x}_{\mathrm{b}}, \mathrm{y}_{\mathrm{b}}\right)$ and $\left(\mathrm{x}_{\mathrm{c}}, \mathrm{y}_{\mathrm{c}}\right)$ of $\mathrm{A}, \mathrm{B}, \mathrm{C}$ respectively. $\mathrm{K}$ is a unknown node move at speed $\mathrm{v}$ with current position $\left(\mathrm{x}_{\mathrm{k}}, \mathrm{y}_{\mathrm{k}}\right)$ and previous position $\left(\mathrm{x}_{\mathrm{k}-1}, \mathrm{y}_{\mathrm{k}-1}\right)$ and we calculate its next position i.e. $\left(\mathrm{x}_{\mathrm{k}+1}, \mathrm{y}_{\mathrm{k}+1}\right)$.

First we calculate the distance from tracking object sensors $\mathrm{D}$ to $\mathrm{A}, \mathrm{B}, \mathrm{C}$ respectively. We assume the distances are $\mathrm{d}_{\mathrm{a}}, \mathrm{d}_{\mathrm{b}}, \mathrm{d}_{\mathrm{c}}$ respectively, i.e.

$$
\begin{aligned}
& \left(x-x_{a}\right)^{2}+\left(y-y_{k}\right)^{2}=d_{a}{ }^{2}, \\
& \left(x-x_{b}\right)^{2}+\left(y-y_{b}\right)^{2}=d_{b}{ }^{2}, \\
& \text { And, }\left(x-x_{c}\right)^{2}+\left(y-y_{c}\right)^{2}=d_{c}{ }^{2}
\end{aligned}
$$

Hence, $D(x, y)^{T}=1 / 2 A^{-1} B$ and from here we can easily found the value of $\mathrm{A}$ and $\mathrm{B}$. To save the energy, among of cluster of sensors, we assume some sensors for cluster head $(\mathrm{CH})$ to trace the location of object and dynamically next cluster head $(\mathrm{CH})$ will be activated. So,

$$
\begin{aligned}
& \mathrm{V}_{\mathrm{A}}=\mathrm{d}_{\mathrm{A}} /\left(\mathrm{t}_{\mathrm{K}}-\left(\mathrm{t}_{\mathrm{k}}-1\right)\right) \\
& \mathrm{V}_{\mathrm{B}}=\mathrm{d}_{\mathrm{B}} /\left(\mathrm{t}_{\mathrm{K}}-\left(\mathrm{t}_{\mathrm{k}}-1\right)\right) \\
& \mathrm{V}_{\mathrm{C}}=\mathrm{d}_{\mathrm{C}} /\left(\mathrm{t}_{\mathrm{K}}-\left(\mathrm{t}_{\mathrm{k}}-1\right)\right)
\end{aligned}
$$

If the object moves along with toward sensor $A$ then it will be $V_{A}, V_{B}$ and $V_{C}$. So, if the next location of object within similar cluster, then previous $\mathrm{CH}$ is activate, other are stand by.Else the next location of object within other clusters, then that cluster head activate and previous will stand by mode. So, CHs measure the current location of sensors within clusters. So that moving object, which location predict by location measure through $\mathrm{CH}$. So, object is traced and our approach saves maximum energy of sensors.

\section{SIMULATION RESULT}

In this paper firstly we compare our general $\mathrm{K}$ mean algorithm and our proposed dynamic $\mathrm{K}$ mean algorithm. As our proposed approach works for large number of cluster with random number of clusters, we take the number of sensor nodes are 400, 800 and 1000. Normal K mean algorithm number of clusters is 4,8,12 where as in our proposed approach; numbers of clusters in dynamic $\mathrm{K}$ mean are 9, 14, and 18 respectively. Firstly we show our Proposed Approach path and Practically generated path.

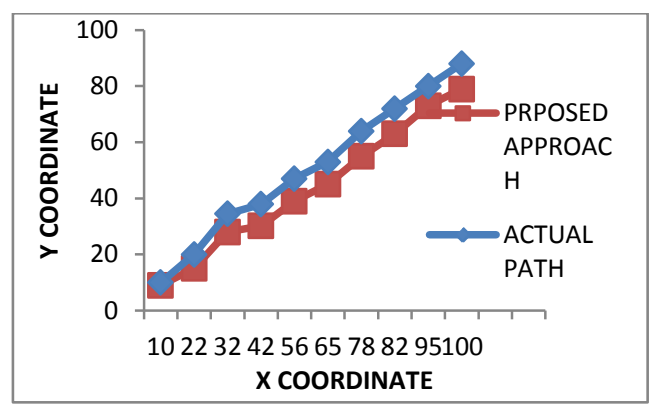

Fig. 1Represent the compare between Proposed Approach path and practically actual path

Here $\mathrm{X}$ axis represents the Time where $\mathrm{Y}$ axis represents the Energy Consumed of Sensor nodes.

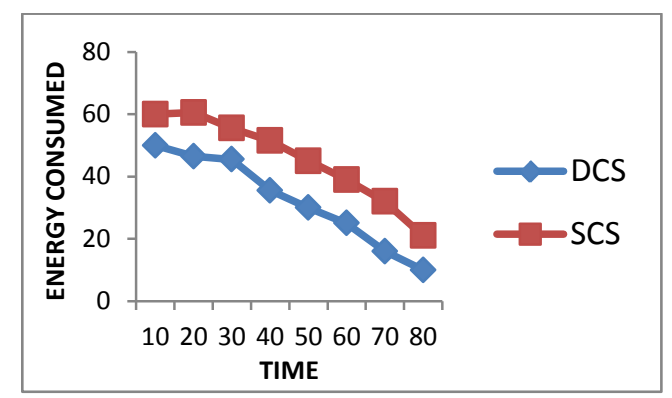

Fig. 2 Proposed Approach of comparison between Dynamically Cluster Sensor (DCS) and Statically Cluster Sensor (SCS) nodes

In this figure, $\mathrm{X}$ axis represents the Number of Sensors Where Y axis represents the Stability of Sensor nodes (ms).

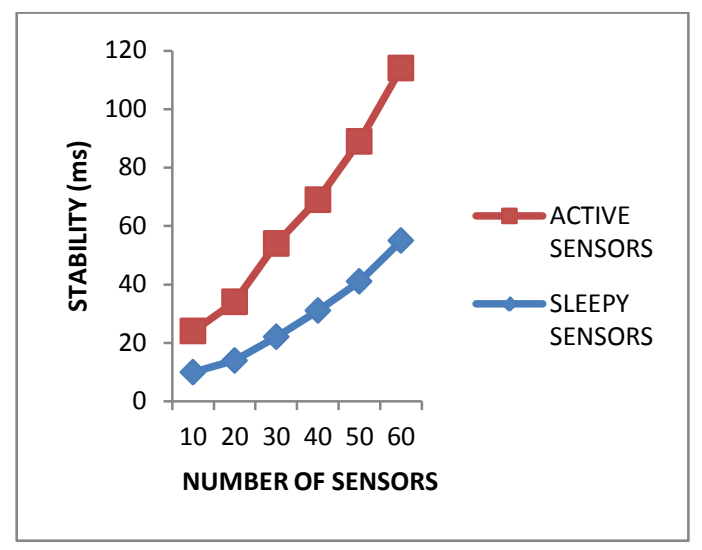

Fig. 3 Stability of Network within the dynamic Cluster in our Proposed Approach.

Our Proposed Approach depicts movable object path within Dynamic Clustering between $\mathrm{X}$ coordinate and $\mathrm{Y}$ coordinate in fig.4.. The Cluster Head (CH) position is as: $\mathrm{CH} 1(20,13)$, CH2(40,13), CH3(60,55), CH4(70,75), CH5(90,90), CH6(100,95), CH7(120,99) where CH1, CH2, CH3, CH4, CH5, CH6, CH7 represent of cluster head 1, 2, 3, 4, 5, 6, 7 respectively. 


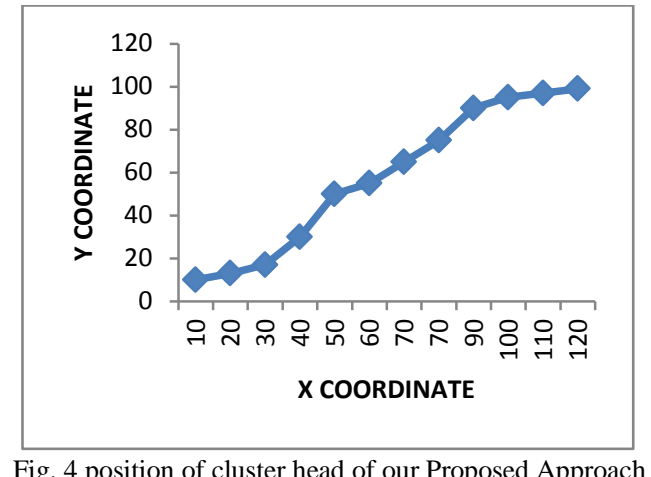

Fig. 4 position of cluster head of our Proposed Approach

\section{ANALYSIS OF RESULT}

According from the fig.1. We can say our proposed Approach path and practically generated path is near about and their error is less than 3 percent. So our Proposed Approach will run successfully to trace a moveable object in dynamic clustering nature. Where as in fig. 2. Suggest that Dynamically Clustering Sensor nodes will run more successfully than Statically Clustering Sensor nodes in our proposed approach. But the fig.3, depicts that Active sensor is more stable than Sleepy Sensors in dynamic clustering approach. So that Active sensors can be used long time to trace an object and lastly in fig.4, describe our Dynamically Proposed Approach Cluster Head (CH) position.

\section{CONCLUSION}

In this paper we show a dynamic clustering technique for object tracing. Our proposed approach works well for unknown data set than traditional $\mathrm{K}$ mean algorithm. The problems of $\mathrm{K}$ mean clustering is to be fixed in advance, so that different data set will appear in same cluster or different have different sizes in a dataset. But our proposed approach overcomes the above problem for search the optimal number. As energy is one of the problems in wireless sensor network in object tracing. Because it is very difficult to replace of battery or sensor nodes within in a cluster. So energy must be saved to stable the network as well as it will be run long time for trace an object. Our proposed approach is also show that a dynamic cluster is formed and a cluster head is active. When an object is traced it shows its position through cluster head into a base station. But if the object is moved the cluster head change its position and new cluster head is assigned. According to the new cluster head position a new cluster has formed. So in this approach maximum energy is saved and whole the networks continue to trace a movable object long time. Our simulation result also indicate that our proposed approach has more network stability and cluster head change its position in respect of current movable object. So cluster head can save energy in maximum mode.

\section{FUTURE WORK}

In future work we can focus to reduce the time complexity in our proposed approach. In this approach, our experiments will be conducted using natural data sets. In this paper we conclude that moveable object is successfully traced by dynamic $\mathrm{K}$ mean clustering algorithm. The work can also extend with multiple movable objects. Our proposed approach can extend to simulate to trace an object with obstruction.

\section{REFERENCES}

[1] S. Prakash Kumar and K. S. Ramaswami, "Efficient Cluster Validation with K-Family Clusters on Quality Assessment", European Journal of Scientific Research, pp.25-36, 2011.

[2] Jiawei Han and Micheline Kamber, "Data Mining: Concepts and Techniques”, Morgan Kaufmann Publishers, second Edition, 2006.

[3] Ye Yingchun, Zhang Laibin, Liang Wei, Yu Dongliang and Wang Zhaohui, "Oil Pipeline Work Conditions Clustering Based on Simulated Annealing K-Means algorithm”, World Congress on Computer Science and Information Engineering, pp. 646-650, 2009.

[4] Khan, S.S., Ahmad, A., "Cluster center initialization algorithm for kmeans clustering”, Pattern Recognition Letter.25, 2004, pp. 12931302.

[5] M. Chu, H. Haussecker and F. Zhao, "Scalable Information-driven Sensor Querying and Routing for Ad Hoc Heterogeneous Sensor Networks", Int'lJ. High Performance Computing Applications, Vol. 16, No. 3, Fall 2002.

[6] J. Liu, J. Liu, J. Reich, P. Cheung and F. Zhao, "Distributed Group Management for Track Initiation and Maintenance in Target Localization Applications," Proc. 2nd Workshop on Information Processing in Sensor Networks(IPSN'03), April 2003.

[7] D. Li, K.Wong, Y. Hu and A. Sayeed, "Detection, Classification, Tracking of Targets in Micro-sensor Networks”, IEEE Signal Processing Magazine, pp. 17-29, March 2002

[8] X. Sheng and Y-H Hu, "Energy Based Acoustic Source Localization", Proc. of 2nd Workshop on Information Processing in Sensor Networks (IPSN’03), April 2003.

[9] F. Zhao, J. Shin and J. Reich, "Information-Driven Dynamic Sensor Collaboration for Tracking Applications”, IEEE Signal Processing Magazine, March 2002.

[10] Y. Xu and W.C. Lee, "On localized prediction for power efficient object tracking in sensor networks", in Proc. 1st Int. Workshop Mobile Distrib. Comput., pp. 434-439, 2003.

[11] G.Y. Jin, X.Y. Lu and M.S. Park, "Dynamic clustering for object tracking in wireless sensor networks", in Proc. 3rd Int. Symp. UCS, Seoul, Korea, pp. 200-209, 2006.

[12] W. Heinzelman, A. Chandrakasan and H. Balakrishnan, "Energyefficient communication protocol for wireless microsensor networks," in Proc.33rd Hawaii Conf. Syst. Sci., pp. 3005-3014, 2000

[13] Y. Zhang, L.T. Yang and J. Chen, "RFID and Sensor NetworksArchitectures, Protocols, Security, and Integrations; CRC”, USA 2010.

[14] N. Patwari, J. Ash, S. Kyperountas, A. Hero and R. Moses, "Correal, $\mathrm{N}$. Locating the nodes: Cooperative localization in wireless sensor networks”, IEEE Signal Proc. Mag., Vol. 22, pp. 54-69, 2005. 